$11-1-2013$

\title{
A Comparison between Biased and Unbiased Estimators in Ordinary Least Squares Regression
}

Ghadban Khalaf

King Khalid University, Saudi Arabia

Follow this and additional works at: http://digitalcommons.wayne.edu/jmasm

Part of the Applied Statistics Commons, Social and Behavioral Sciences Commons, and the Statistical Theory Commons

\section{Recommended Citation}

Khalaf, Ghadban (2013) "A Comparison between Biased and Unbiased Estimators in Ordinary Least Squares Regression," Journal of Modern Applied Statistical Methods: Vol. 12 : Iss. 2 , Article 17.

DOI: $10.22237 /$ jmasm/1383279360

Available at: http://digitalcommons.wayne.edu/jmasm/vol12/iss2/17

This Regular Article is brought to you for free and open access by the Open Access Journals at DigitalCommons@WayneState. It has been accepted for inclusion in Journal of Modern Applied Statistical Methods by an authorized editor of DigitalCommons@WayneState. 


\section{A Comparison between Biased and Unbiased Estimators in Ordinary Least Squares Regression}

\section{Ghadban Khalaf}

King Khalid University

Saudi Arabia

During the past years, different kinds of estimators have been proposed as alternatives to the Ordinary Least Squares (OLS) estimator for the estimation of the regression coefficients in the presence of multicollinearity. In the general linear regression model, $\vec{Y}=X \vec{\beta}+\vec{e}$, it is known that multicollinearity makes statistical inference difficult and may even seriously distort the inference. Ridge regression, as viewed here, defines a class of estimators of $\vec{\beta}$ indexed by a scalar parameter $k$. Two methods of specifying $k$ are proposed and evaluated in terms of Mean Square Error (MSE) by simulation techniques. A comparison is made with other ridge-type estimators evaluated elsewhere. The estimated MSE of the suggested estimators are lower than other estimators of the ridge parameter and the $O L S$ estimator.

Keywords: $\quad$ OLS estimator, linear regression, multicollinearity, ridge regression, Monte Carlo simulation.

\section{Introduction}

Consider the multiple linear regression model

$$
\vec{Y}=X \vec{\beta}+\vec{e}
$$

where $\vec{Y}$ is an $(n \times 1)$ response vector, $X$ is a fixed $(n \times p)$ matrix of independent variables of rank $p, \vec{\beta}$ is the unknown $(p \times 1)$ parameter vector of regression coefficients and, finally, $\vec{e}$ is an $(n \times 1)$ vector of uncorrelated errors with mean zero and common unknown variance $\sigma^{2}$. If $X^{\prime} X$ is nonsingular, the OLS estimator for $\vec{\beta}$ is given by

Ghadban Khalaf is an Associate Professor in the Department of Mathematics. 


\section{A COMPARISON BETWEEN BIASED AND UNBIASED ESTIMATORS}

$$
\hat{\beta}=\left(X^{\prime} X\right)^{-1} X^{\prime} \vec{Y}
$$

For orthogonal data, the OLS estimator in the linear regression model is strongly efficient. But in the presence of multicollinearity, the OLS efficiency can be reduced and hence an improvement upon it would be necessary and desirable.

The term multicollinearity is used to denote the presence of linear relationships, or near linear relationships, among explanatory variables. If the explanatory variables are perfectly linearly correlated, that is, if the correlation coefficient for these variables is equal to unity, then the parameters become indeterminate; i.e, it is impossible to obtain numerical values for each parameter separately and the method of lease squares breaks down. Conversely, if the correlation coefficient for the explanatory variables is equal to zero, then the variables are called orthogonal and there are no problems concerning the estimates of the coefficients.

When multicollinearity occurs, the least squares estimates are still unbiased and efficient but the problem is that; the estimated standard error $S_{\hat{\beta}_{i}}$ for the

coefficient $\hat{\beta}_{i}$ become infinitely large; i.e, the standard error tends to be larger than it would be in the absence of multicollinearity and when $S_{\hat{\beta}_{i}}$ is larger than it would be, then the $t$ - value for testing the significance of $\beta_{i}$ is smaller than it should be. Thus one is likely to conclude that a variable $X_{i}$ is not important in the relationship when it real1y is.

To solve the problem of multicollinearity, there is no single solution that will eliminate multicollinearity altogether. One common procedure is to select the independent variable most seriously involved in the multicollinearity and remove it from the model. This procedure often improves the standard error of the remaining coefficients and may make formerly insignificant variables significant, since the elimination of a variable reduces any multicollinearity caused by it. The difficulty with this approach is that the model now may not correctly represent the population relationship and all estimated coefficients would contain a population specification.

The procedure of increasing the sample size is sometimes recommended as another suggested procedure to solve the problem of multicollinearity. In fact this method improves the precision of an estimator and hence reduces the adverse effects of multicollinearity.

Hoerl and Kennard (1970) suggested a new technique to overcome the problem of multicollinearity. This technique is called ridge regression. Ridge 


\section{GHADBAN KHALAF}

regression is a variant of ordinary multiple linear regression whose goal is to circumvent the predictors collinearity. It gives up the least squares as a method for estimating the parameters of the model and focuses instead of the $X^{\prime} X$ matrix. This matrix will be artificially modified so as to make its determinant appreciably different from zero. This is accomplished by adding a small positive quantity, say $k(k>0)$, to each of the diagonal elements of the matrix $X^{\prime} X$ before inverting it for least squares estimation. The resulting estimator is given by

$$
\overrightarrow{\hat{\beta}}(k)=\left(X^{\prime} X+k I_{p}\right)^{-1} X^{\prime} \vec{Y}, \quad k>0
$$

which coincides with the OLS estimator, defined by (2), when $k=0$. The resulting estimator will be biased, but have smaller variances than $\overrightarrow{\hat{\beta}}$. This is precisely what the ridge regression estimator we study can accomplish.

The plan of this paper is as follows: Section 2 presents the proposed estimators included in the study; a novel feature is our proposed ridge estimator which, as we shall see presently, has lower MSE. Section 3 is described the simulation technique that we have adopted in our study to evaluate the performance of the new values of the ridge parameter we suggest. The results of the simulation study, which appear in the tables, are presented in Section 4. Finally, Section 5 contains summary and conclusions.

\section{The Proposed Estimators}

With the ridge estimator method, there arises the problem of determining an optimal value of $k$. With a good choice of $k$, one might hope to improve on the OLS estimator for every coefficient.

Hoerl, Kennard and Baldwin (1975) showed, through simulation, that the use of ridge estimator with the following biasing parameter

$$
\hat{k}=\frac{p \hat{\sigma}^{2}}{\sum_{i=1}^{p} \hat{\beta}_{i}^{2}}
$$

implies that $\operatorname{MSE}(\overrightarrow{\hat{\beta}}(k))<\operatorname{MSE}(\overrightarrow{\hat{\beta}})$, where $p$ denotes the number of parameters (excluding the intercept) and $\hat{\sigma}^{2}$ is the usual unbiased estimate of $\sigma^{2}$, defined by; 


\section{A COMPARISON BETWEEN BIASED AND UNBIASED ESTIMATORS}

$$
\hat{\sigma}^{2}=\sum_{i=1}^{n} e_{i}^{2} /(n-p-1)
$$

They showed that the probability of a smaller MSE using (4) increases with the number of parameters $p$. We will use the acronym $H K B$ for the estimator (4).

Khalaf and Shukur (2005) suggested a modification of Hoerl and Kennard (1970) given by;

$$
\hat{k}=\frac{t_{\max } \hat{\sigma}^{2}}{(n-p) \hat{\sigma}^{2}+t_{\max } \hat{\beta}_{\max }^{2}}
$$

which guaranteed lower MSE, where $t_{\max }$ is the maximum eigenvalue of $X^{\prime} X$ matrix. For this estimator we will use the acronym $K S$.

From the estimators (4) and (5), we suggest as a modification of $H K B$ and $K S$ by multiplying them by the amount;

$$
\frac{\frac{1}{2}\left(t_{\max }+t_{\min }\right)}{\sum_{i=1}^{p}\left|\hat{\beta}_{i}\right|}=\frac{t_{\max }+t_{\min }}{2 \sum_{i=1}^{p}\left|\hat{\beta}_{i}\right|},
$$

where $t_{\min }$ is the minimum eigenvalue of the matrix $X^{\prime} X$. This leads to the following estimators;

$$
\begin{gathered}
\hat{k}_{1}=\frac{\left(t_{\max }+t_{\min }\right)}{2 \sum_{i=1}^{p}\left|\hat{\beta}_{i}\right|} \cdot \frac{p \hat{\sigma}^{2}}{\sum_{i=1}^{p} \hat{\beta}_{i}^{2}} \\
\hat{k}_{2}=\frac{\left(t_{\max }+t_{\min }\right) t_{\max } \hat{\sigma}^{2}}{2 \sum_{i=1}^{p}\left|\hat{\beta}_{i}\right|\left((n-p) \hat{\sigma}^{2}+t_{\max } \hat{\beta}_{\max }^{2}\right)} .
\end{gathered}
$$

For our two suggested estimators, defined by (6) and (7), we use the acronym $K_{1}$ and $K_{2}$, respectively. 


\section{GHADBAN KHALAF}

\section{The Simulation Study}

A simulation study was conducted in order to draw conclusions about the performance of our suggested estimators relative to $H K B, K S$ and the OLS estimator. To achieve different degree of collinearity, following Kibria (2003), the explanatory variables are generated by using the following equation;

$$
x_{i j}=\left(1-\rho^{2}\right)^{1 / 2} z_{i j}+\rho z_{i p}, \quad i=1,2, \ldots, n . \quad j=1,2, \ldots, p
$$

where $Z_{i j}$ are independent standard normal distribution, $p$ is the number of the explanatory variables and ${ }^{\rho}$ is specified so that the correlation between any two explanatory variables is given by $\rho^{2}$. Three different sets of correlation are considered according to the value of $\rho=0.85,0.95$ and 0.99 . The $n$ observations for the dependent variable are determined by the following equation;

$$
y_{i}=\beta_{0}+\beta_{1} x_{i 1}+\beta_{2} x_{i 2}+\ldots+\beta_{p} x_{i p}+e_{i}, \quad i=1,2, \ldots, n
$$

where $e_{i}$ are i.i.d pseudo-random numbers. In this study, $\beta_{0}$ is taken to be zero and the term $e_{i}$ is generated from each of the following distributions: $N(0,1)$, $T(3), T(7)$ and $F(3,11)$. The parameters values are chosen so that $\sum_{i=1}^{p} \beta_{i}^{2}=1$, which is a common restriction in simulation studies (see Muniz and Kibria (2009)).

The other factors we chose to vary is the sample size and the number of regressions. We generate models consisting of 25, 50, 100 and 150 observations and with 2 and 4 explanatory variables. It is noted from the results of the previous simulation studies (see Khalaf and Shukur (2005), Alkhamisi and Shukur (2008) and Khalaf (2011)) that increasing the number of regressor and using non-normal pseudo random numbers to generate $e_{i}$ leads to a higher estimated MSE, while increasing the sample size leads to a lower estimated MSE.

The criterion proposed here for measuring the goodness of an estimator is the MSE using the following formula;

$$
\operatorname{MSE}\left(\overrightarrow{\hat{\beta}}_{r}\right)=\frac{1}{5000} \sum\left(\overrightarrow{\hat{\beta}}_{r}-\vec{\beta}\right)^{\prime}\left(\overrightarrow{\hat{\beta}}_{r}-\vec{\beta}\right)
$$




\section{A COMPARISON BETWEEN BIASED AND UNBIASED ESTIMATORS}

where $\overrightarrow{\hat{\beta}}_{r}$ is the estimator of $\vec{\beta}$ obtained from the OLS estimator or from the ridge estimator for different estimated values of $k$ considered for comparison reasons and, finally, 5000 is the number of replicates used in the Monte Carlo simulation.

\section{The Simulation Results}

Tables 1 - 6 below, present the output from the Monte Carlo experiment concerning properties of the different methods that used to choose the ridge parameter $k$. The results showed that the estimated MSE is affected by all factors we choose to vary in the design of experiment. It is also noted that the higher the degree of correlation the higher estimated MSE, but this increase is much greater for the OLS than the ridge regression estimator. The distribution of the error term and the number of explanatory variables having a different impact on the estimators.

Table 1. Estimated MSE when $\rho=0.85$ and $p=2$.

\begin{tabular}{|c|c|c|c|c|c|}
\hline & OLS & $H K B$ & $K S$ & $K_{1}$ & $K_{2}$ \\
\hline \multicolumn{6}{|l|}{$N(0,1)$} \\
\hline 25 & 0.238 & 0.181 & 0.190 & 0.243 & 0.181 \\
\hline 50 & 0.111 & 0.093 & 0.097 & 0.255 & 0.176 \\
\hline 100 & 0.057 & 0.051 & 0.053 & 0.266 & 0.178 \\
\hline 150 & 0.034 & 0.032 & 0.032 & 0.282 & 0.184 \\
\hline \multicolumn{6}{|l|}{$T(3)$} \\
\hline 25 & 2.259 & 0.957 & 1.325 & 0.506 & 0.497 \\
\hline 50 & 1.219 & 0.602 & 0.896 & 0.521 & 0.364 \\
\hline 100 & 0.531 & 0.312 & 0.445 & 0.588 & 0.329 \\
\hline 150 & 0.473 & 0.261 & 0.414 & 0.632 & 0.351 \\
\hline \multicolumn{6}{|l|}{$T(7)$} \\
\hline 25 & 0.350 & 0.248 & 0.266 & 0.304 & 0.218 \\
\hline 50 & 0.169 & 0.135 & 0.143 & 0.324 & 0.212 \\
\hline 100 & 0.076 & 0.067 & 0.069 & 0.352 & 0.220 \\
\hline 150 & 0.053 & 0.048 & 0.049 & 0.367 & 0.224 \\
\hline \multicolumn{6}{|l|}{$F(3,11)$} \\
\hline 25 & 0.853 & 0.502 & 0.584 & 0.383 & 0.289 \\
\hline 50 & 0.391 & 0.261 & 0.309 & 0.429 & 0.236 \\
\hline 100 & 0.178 & 0.139 & 0.157 & 0.481 & 0.276 \\
\hline 150 & 0.126 & 0.104 & 0.115 & 0.520 & 0.290 \\
\hline
\end{tabular}




\section{GHADBAN KHALAF}

Table 2. Estimated MSE when $\rho=0.85$ and $p=4$.

\begin{tabular}{|c|c|c|c|c|c|}
\hline & OLS & $H K B$ & $K S$ & $K_{1}$ & $K_{2}$ \\
\hline \multicolumn{6}{|l|}{$N(0,1)$} \\
\hline 25 & 0.796 & 0.549 & 0.572 & 0.187 & 0.159 \\
\hline 50 & 0.334 & 0.255 & 0.268 & 0.216 & 0.162 \\
\hline 100 & 0.156 & 0.131 & 0.137 & 0.257 & 0.182 \\
\hline 150 & 0.103 & 0.090 & 0.093 & 0.284 & 0.196 \\
\hline \multicolumn{6}{|l|}{$T(3)$} \\
\hline 25 & 7.387 & 3.781 & 4.330 & 1.049 & 1.205 \\
\hline 50 & 6.222 & 2.961 & 4.179 & 1.073 & 1.622 \\
\hline 100 & 1.685 & 0.969 & 1.318 & 0.509 & 0.409 \\
\hline 150 & 1.240 & 0.754 & 1.018 & 0.551 & 0.332 \\
\hline \multicolumn{6}{|l|}{$T(7)$} \\
\hline 25 & 1.159 & 0.730 & 0.776 & 0.212 & 0.178 \\
\hline 50 & 0.504 & 0.362 & 0.389 & 0.255 & 0.184 \\
\hline 100 & 0.235 & 0.188 & 0.200 & 0.323 & 0.218 \\
\hline 150 & 0.152 & 0.127 & 0.135 & 0.353 & 0.231 \\
\hline \multicolumn{6}{|l|}{$F(3,11)$} \\
\hline 25 & 2.667 & 1.446 & 1.601 & 0.338 & 0.328 \\
\hline 50 & 1.130 & 0.699 & 0.805 & 0.316 & 0.226 \\
\hline 100 & 0.578 & 0.402 & 0.468 & 0.415 & 0.263 \\
\hline 150 & 0.362 & 0.271 & 0.311 & 0.467 & 0.282 \\
\hline
\end{tabular}

Table 3. Estimated MSE when $\rho=0.95$ and $p=2$.

\begin{tabular}{|c|c|c|c|c|c|}
\hline & OLS & $H K B$ & $K S$ & $K_{1}$ & $K_{2}$ \\
\hline \multicolumn{6}{|l|}{$N(0,1)$} \\
\hline 25 & 0.705 & 0.427 & 0.450 & 0.193 & 0.147 \\
\hline 50 & 0.353 & 0.250 & 0.265 & 0.193 & 0.134 \\
\hline 100 & 0.168 & 0.133 & 0.140 & 0.220 & 0.145 \\
\hline 150 & 0.114 & 0.095 & 0.010 & 0.231 & 0.149 \\
\hline \multicolumn{6}{|l|}{$T(3)$} \\
\hline 25 & 7.899 & 3.010 & 3.580 & 1.501 & 1.725 \\
\hline 50 & 5.575 & 2.137 & 2.969 & 0.988 & 1.256 \\
\hline 100 & 1.703 & 0.789 & 1.152 & 0.486 & 0.275 \\
\hline 150 & 1.283 & 0.655 & 0.959 & 0.541 & 0.299 \\
\hline \multicolumn{6}{|l|}{$T(7)$} \\
\hline 25 & 1.174 & 0.670 & 0.718 & 0.241 & 0.186 \\
\hline 50 & 0.528 & 0.340 & 0.371 & 0.249 & 0.164 \\
\hline 100 & 0.250 & 0.185 & 0.200 & 0.287 & 0.177 \\
\hline 150 & 0.161 & 0.127 & 0.137 & 0.311 & 0.187 \\
\hline \multicolumn{6}{|l|}{$F(3,11)$} \\
\hline 25 & 2.556 & 1.223 & 1.372 & 0.401 & 0.343 \\
\hline 50 & 1.167 & 0.623 & 0.738 & 0.336 & 0.215 \\
\hline 100 & 0.566 & 0.346 & 0.419 & 0.397 & 0.224 \\
\hline 150 & 0.378 & 0.251 & 0.300 & 0.444 & 0.244 \\
\hline
\end{tabular}




\section{A COMPARISON BETWEEN BIASED AND UNBIASED ESTIMATORS}

Table 4. Estimated MSE when $\rho=0.95$ and $p=4$.

\begin{tabular}{lcccccc}
\hline \multicolumn{1}{c}{} & OLS & HKB & KS & $\boldsymbol{K}_{1}$ & $\boldsymbol{K}_{\mathbf{2}}$ \\
\hline $\boldsymbol{N}(\mathbf{0}, \mathbf{1})$ & & & & & \\
& 25 & 2.356 & 1.308 & 1.351 & 0.154 & 0.145 \\
& 50 & 1.057 & 0.673 & 0.705 & 0.125 & 0.099 \\
& 100 & 0.359 & 0.251 & 0.266 & 0.120 & 0.088 \\
& 150 & 0.323 & 0.245 & 0.258 & 0.194 & 0.138 \\
\hline $\boldsymbol{T ( 3 )}$ & & & & & \\
& 25 & 18.886 & 8.484 & 9.026 & 2.145 & 2.299 \\
& 50 & 14.573 & 7.274 & 8.218 & 1.939 & 2.315 \\
& 100 & 4.122 & 2.079 & 2.527 & 0.284 & 0.194 \\
& 150 & 3.390 & 1.815 & 2.266 & 0.422 & 0.306 \\
\hline $\boldsymbol{T ( 7 )}$ & & & & & \\
& 25 & 3.716 & 1.996 & 2.068 & 0.228 & 0.221 \\
& 50 & 1.502 & 0.892 & 0.948 & 0.146 & 0.114 \\
& 100 & 0.745 & 0.495 & 0.534 & 0.199 & 0.139 \\
& 150 & 0.478 & 0.341 & 0.368 & 0.239 & 0.162 \\
\hline $\boldsymbol{F ( 3 , 1 1 )}$ & & & & & \\
& 25 & 8.220 & 4.148 & 4.334 & 0.776 & 0.796 \\
& 50 & 3.578 & 1.882 & 2.064 & 0.206 & 0.171 \\
& 100 & 1.755 & 1.034 & 1.170 & 0.248 & 0.164 \\
& 150 & 1.180 & 0.741 & 0.849 & 0.309 & 0.195 \\
\hline
\end{tabular}

Table 5. Estimated MSE when $\rho=0.99$ and $p=2$.

\begin{tabular}{lcccccc}
\hline \multicolumn{1}{c}{} & OLS & HKB & KS & $\boldsymbol{K}_{\mathbf{1}}$ & $\boldsymbol{K}_{\mathbf{2}}$ \\
\hline $\boldsymbol{N}(\mathbf{0}, \mathbf{1})$ & & & & & \\
& 25 & 4.050 & 1.850 & 1.905 & 0.349 & 0.331 \\
& 50 & 1.776 & 0.884 & 0.931 & 0.133 & 0.099 \\
& 100 & 0.913 & 0.533 & 0.568 & 0.138 & 0.091 \\
& 150 & 0.572 & 0.358 & 0.385 & 0.155 & 0.099 \\
\hline $\boldsymbol{T ( 3 )}$ & & & & & \\
& 25 & 43.786 & 15.618 & 16.407 & 12.046 & 12.512 \\
& 50 & 21.736 & 7.673 & 8.510 & 3.155 & 3.377 \\
& 100 & 8.794 & 3.602 & 4.217 & 0.481 & 0.399 \\
& 150 & 7.046 & 2.461 & 3.274 & 0.362 & 0.231 \\
\hline $\boldsymbol{T ( 7 )}$ & & & & & \\
& 25 & 6.108 & 2.657 & 2.745 & 0.561 & 0.544 \\
& 50 & 2.623 & 1.192 & 1.274 & 0.171 & 0.124 \\
& 100 & 1.370 & 0.732 & 0.797 & 0.178 & 0.111 \\
& 150 & 0.865 & 0.502 & 0.551 & 0.204 & 0.123 \\
\hline $\boldsymbol{F ( 3 , 1 1 )}$ & & & & & 1.438 \\
& 25 & 12.863 & 4.822 & 5.037 & 1.421 & 0.296 \\
& 50 & 6.402 & 2.550 & 2.779 & 0.343 & 0.152 \\
& 100 & 3.329 & 1.508 & 1.715 & 0.246 & 0.151 \\
\hline & 150 & 1.901 & 0.899 & 1.060 & 0.279 & \\
\hline
\end{tabular}




\section{GHADBAN KHALAF}

Table 6. Estimated MSE when $\rho=0.99$ and $p=4$.

\begin{tabular}{|c|c|c|c|c|c|}
\hline & OLS & $H K B$ & $K S$ & $K_{1}$ & $K_{2}$ \\
\hline \multicolumn{6}{|l|}{$N(0,1)$} \\
\hline 25 & 13.319 & 6.484 & 6.547 & 0.971 & 0.981 \\
\hline 50 & 6.095 & 3.078 & 3.141 & 0.109 & 0.107 \\
\hline 100 & 2.708 & 1.466 & 1.520 & 0.061 & 0.050 \\
\hline 150 & 1.720 & 0.990 & 1.036 & 0.076 & 0.057 \\
\hline \multicolumn{6}{|l|}{$T(3)$} \\
\hline 25 & 169.385 & 72.238 & 73.397 & 58.482 & 59.442 \\
\hline 50 & 65.170 & 33.982 & 34.732 & 15.466 & 15.685 \\
\hline 100 & 30.913 & 15.077 & 15.913 & 2.328 & 2.448 \\
\hline 150 & 19.922 & 8.885 & 9.738 & 0.505 & 0.556 \\
\hline \multicolumn{6}{|l|}{$T(7)$} \\
\hline 25 & 19.789 & 9.337 & 9.473 & 1.739 & 1.756 \\
\hline 50 & 8.782 & 4.342 & 4.442 & 0.230 & 0.229 \\
\hline 100 & 4.068 & 2.152 & 2.240 & 0.077 & 0.063 \\
\hline 150 & 2.550 & 1.390 & 1.467 & 0.086 & 0.062 \\
\hline \multicolumn{6}{|l|}{$F(3,11)$} \\
\hline 25 & 44.422 & 20.834 & 21.062 & 7.010 & 7.089 \\
\hline 50 & 21.347 & 9.485 & 9.785 & 1.073 & 1.131 \\
\hline 100 & 9.172 & 4.498 & 4.730 & 0.145 & 0.131 \\
\hline 150 & 6.178 & 3.077 & 3.303 & 0.114 & 0.085 \\
\hline
\end{tabular}

For non-normal error term in combination with $\rho=0.95$ and $\rho=0.99$ leads to a larger estimated MSE for the OLS estimator and the ridge parameter, especially when $n$ is small, but when the sample size increases the estimated MSE of the suggested ridge parameters, namely $K_{1}$ and $K_{2}$ decreases substantially.

The performance of $K_{1}$ and $K_{2}$ is well for all cases when the error term is distributed as a normal and, when $n$ is greater than 25 and the error term in nonnormal .

When $n$ is greater than 25, the modified ridge parameter performance, defined by (6) and (7), is much better than the estimators $H K B, K S$ and the OLS, where $K_{2}$ has a low estimated MSE when the number of regressor equals 4 .

\section{Summary and Conclusions}

In multiple linear regression, the effect of non-orthogonality of the explanatory variables is to pull the least squares estimates of the regression coefficients away from the true coefficients, $\vec{\beta}$, that one is trying to estimate. The coefficients can 


\section{A COMPARISON BETWEEN BIASED AND UNBIASED ESTIMATORS}

be both too large in absolute value and incorrect with respect to sign. Furthermore, the variance and the covariance of the OLS tend to become too large.

A slight movement away from this point can give completely different estimates of the coefficients. This is accomplished by adding a small positive quantity, $k$, to each of the diagonal elements of the matrix $X^{\prime} X$. The resulting estimator is called the ridge estimator, suggested by Hoerl and Kennard (1970) and given by (3).

Several procedure for constructing ridge estimators have been proposed in the literature. These procedures were aiming at a rule (or algorithm) for selecting the constant $k$ in equation (3). In fact, the best method of estimating $k$ is an unsolved problem and there is no constant value of $k$ that is certain to yield an estimator which is uniformly better (in terms of MSE) than the OLS in all cases.

By means of Monte Carlo simulations two suggested ridge parameters were evaluated and the result were compared with ridge parameters evaluated by Hoerl et. al (1975) and Khalaf and Shukur (2005). The estimator HKB performed well in this study. It appears to outperform $K S$ when $\rho$ is small and the sample size is greater than 25. The suggested estimators $K_{1}$ and $K_{2}$ performs well in our simulation. They appeared to offer an opportunity for large reduction in MSE when $p=2$ and the error term in normally distributed. For non-normal error term the versions of the ridge parameter has a lower estimated MSE when the sample size is greater than 25. $K_{2}$ is always minimizes the estimated MSE when the error term in normally distributed.

\section{References}

Alkhamisi, M., \& Shukur, G. (2008). Developing Ridge Parameters for SUR Model. Communication in Statistics- Theory and Methods, 37, 544-564.

Hoerl, A. E., \& Kennard, R. W. (1970). Ridge Regression: Biased Estimation for non-orthogonal Problems. Technometrics, Vol. 12, 55 - 67.

Hoerl, A. E., Kennard, R. W., \& Baldwin, K. F. (1975). Ridge Regression: some Simulation. Communications in Statistics- Theory and Methods, 4, 105 124.

Khalaf, G. (2011). Suggested Ridge Regression Estimators Under Multicollinearity. Journal of Natural and Applied Sciences, University of Aden, Vol. 15, $170-193$. 


\section{GHADBAN KHALAF}

Khalaf, G., \& Shukur, G. (2005). Choosing Ridge Parameters for Regression Problems. Communication in Statistics - Theory and Methods, 34, 1177 - 1182.

Kibria, B. M. G. (2003). Performance of some New Ridge Regression Estimators. Communication in Statistics- Theory and Methods, 32, 419-435.

Muniz, G., \& Kibria, B. M. G. (2009). On some Ridge Regression Estimators: An Empirical Comparisons. Communications in Statistics-Simulation and Computation, 38, 621 - 630. 